\title{
Bispectrum speckle interferometry of the massive protostellar outflow source AFGL 2591
}

\author{
T. Preibisch ${ }^{1}$, Y. Y. Balega ${ }^{2}$, D. Schertl ${ }^{1}$, and G. Weigelt ${ }^{1}$ \\ 1 Max-Planck-Institut für Radioastronomie, Auf dem Hügel 69, 53121 Bonn, Germany \\ 2 Special Astrophysical Observatory, Nizhnij Arkhyz, Zelenchuk region, Karachai-Cherkesia, 357147, Russia
}

Received 10 June 2003 / Accepted 28 August 2003

\begin{abstract}
We present bispectrum speckle interferometry of the massive protostellar object AFGL 2591 in the near-infrared $K$-band. Our reconstructed image of the outflow cavity of AFGL 2591 has a resolution of 170 mas, corresponding to physical scales of $\sim 170 \mathrm{AU}$ at the distance of the object, and shows the loops which extend from the bright, compact source in unprecedented detail. The central source is clearly resolved and has an uniform-disk diameter of $\sim 40$ mas (40 AU). We use 2D radiation transfer simulations to show that the resolved structure probably corresponds to the inner rim of a geometrically thick circumstellar disk or envelope at the dust sublimation radius. Our image also reveals a structure that might represent an edge-on circumstellar disk around one of the other young stellar objects near AFGL 2591.
\end{abstract}

Key words. techniques: interferometric - stars: individual: AFGL 2591 - stars: formation - stars: winds, outflows

\section{Introduction}

Jets and outflows from young stellar objects are widely recognized as an essential ingredient of the star formation process (for a recent review see e.g. Reipurth \& Bally 2001). In particular, they are believed to contribute to the removal of excess angular momentum from accreted matter and to disperse infalling circumstellar envelopes. Many massive protostars drive strong winds, very energetic outflows, and high velocity jets that disrupt their circumstellar surroundings. The jets and outflows from high-mass protostars generally display a considerably more complex structure and often appear to be less well collimated than outflows from low-mass young stellar objects. Despite their key role in star formation, the origin of the flows themselves remains elusive, although it is believed that their generation involves the simultaneous action of magnetic and centrifugal forces in a rotating star/disk system. It is currently unknown whether the jets originate from the star or from the disk. The different theoretical models have not been tested observationally, since the process is believed to occur on very small spatial scales. The origin of the jets and outflows therefore represents one of the fundamental open problems in star formation.

High spatial resolution imaging of the inner circumstellar environment in young stellar objects can shed light onto the origin of the flows and the mechanism responsible for the initial collimation of the beams. Studies with the HST, adaptive optics, and speckle interferometry have recently yielded

Send offprint requests to: T. Preibisch, e-mail: preib@mpifr-bonn.mpg.de important information on the inner circumstellar environment of many young stellar objects (e.g. Stapelfeldt et al. 1998; Brandner et al. 2000) and have revealed spectacular manifestations of the interaction of the jets and outflows with the surrounding material (e.g. Dougados et al. 2000). Bispectrum speckle interferometry has revealed inner circumstellar structures of very complex morphology around several massive and intermediate-mass young stellar objects (e.g. Schertl et al. 2000; Weigelt et al. 2002a,b; Preibisch et al. 2001, 2002). This was the motivation of our speckle interferometric study of the young stellar object AFGL 2591 presented in this paper.

AFGL 2591 is an optically invisible infrared source in a relatively isolated massive star formation region in Cygnus. It represents one of the most massive young stellar objects which are bright at near-infrared wavelengths. The distance to AFGL 2591 is highly uncertain; following the arguments summarized in van der Tak et al. (1999) we use here a value of $1 \mathrm{kpc}$. The luminosity of the source is then $\sim 2 \times 10^{4} L_{\odot}$, suggesting a mass of about $10-15 M_{\odot}$ for the young stellar object. AFGL 2591 drives a powerful bipolar molecular outflow (Bally \& Lada 1983; Lada et al. 1984) which has an extent of $\sim 1.5 \mathrm{pc}$ in east-west direction $\left(\mathrm{PA} \sim 270^{\circ}\right)$ and a dynamical age of $2 \times 10^{4} \mathrm{yrs}$. The near-infrared continuum emission (see e.g. the $K$-band image in Hodappp 1994) shows a well defined conical structure with a bright, compact infrared source centered at the apex. This morphology suggests the presence of disk-like obscuring material in a plane perpendicular to the outflow direction. The disk is tilted in such a way that its polar region to the West is nearer to the Sun; therefore, the western region is less obscured by the disk than the eastern region. The strong 
extinction in the circumstellar envelope and disk blocks our view of the central protostar at optical wavelengths and even in the near-infrared $J$ band.

Near-infrared imaging and polarimetry by Tamura et al. (1991) and Minchin et al. (1991) revealed two loops of nebulosity aligned with the high-velocity blue-shifted outflow to the West of the infrared point source. The shape and polarization of the structures suggested they are reflection nebulosity. The polarization vector pattern is consistent with AFGL 2591 as the sole illuminator of the outflow region. The two loops were interpreted as the result of two discrete mass outbursts from AFGL 2591.

Poetzel et al. (1992) detected several Herbig-Haro objects $23^{\prime \prime}$ to $35^{\prime \prime}$ to the West of AFGL 2591, a clear indication of jetlike flows from the central object. Tamura \& Yamashita (1992) presented near-infrared long-slit spectrophotometric measurements of AFGL 2591. They detected strong emission in the $v=1-0 \mathrm{~S}(1)$ ro-vibrational line of molecular hydrogen at $2.12 \mu \mathrm{m}$, which is a convenient tracer of low- to moderatevelocity shocks (e.g. Smith 1993; Eislöffel et al. 2000). The $\mathrm{H}_{2}$ shock emission extends over $90^{\prime \prime}(0.9 \mathrm{pc})$ in east-west direction and is composed of several knots. It marks those regions where the outflowing material interacts with the surrounding molecular cloud.

The circumstellar environment of AFGL 2591 has been studied in detail by van der Tak et al. (1999) with millimeter interferometry, single-dish submillimeter observations, and nearinfrared absorption line observations. These data were used to constrain the physical and kinematical structure of the dust and gas on scales of $\sim 100$ AU to $\sim 30000$ AU. Their results can be summarized as follows: AFGL 2591 is probably an early Btype star surrounded by a large $(R \sim 30000 \mathrm{AU})$ envelope with a total extinction of $A_{V} \sim 100 \mathrm{mag}$. The central part of the envelope seems to contain a compact, dense structure; the geometry of this inner structure is not constrained by their data, but one possibility is an optically thick circumstellar disk or torus with a radius of about $30 \mathrm{AU}$. The total circumstellar mass derived from radio continuum measurements is $\sim 42 M_{\odot}$. In the bipolar outflow cavities, molecular gas is flowing outwards at velocities of about $20 \mathrm{~km} \mathrm{~s}^{-1}$. The star drives an ionized wind with a velocity of about $500 \mathrm{~km} \mathrm{~s}^{-1}$; at the edge of the cavity this wind entrains material from the envelope and accelerates it to flow velocities of about $200 \mathrm{~km} \mathrm{~s}^{-1}$. The outflow axis is inclined by about $30^{\circ}$ from the line-of-sight.

An extensive survey of the literature indicates that all published imaging studies of AFGL 2591 were seeing-limited. The highest spatial resolution image that has so far been presented for AFGL 2591 seems to be the JHK image obtained with the Gemini North telescope, which can be found at the Gemini website $^{1}$. It was taken with the Near Infrared Imager during its commissioning phase. As described on the www site, the images have a resolution of $0.4^{\prime \prime}$ and reveal remarkable details in the nebula around AFGL 2591. The nebulosity to the West of the bright central source is resolved into a series of four distinct rings. To the East of the central source, the Gemini $K$-band image reveals faint traces of gas, which indicate interactions

\footnotetext{
${ }^{1}$ http://www.gemini.edu/gallery/science/g0136.html
}

Table 1. Observational parameters. $N_{\mathrm{T}}$ and $N_{\mathrm{R}}$ are the numbers of speckle interferograms of AFGL 2591 and the reference star, respectively. $T$ is the exposure time per frame and $\mathrm{S}$ is the Seeing (FWHM).

\begin{tabular}{crcrrr}
\hline \hline $\begin{array}{c}\text { Date } \\
\text { Sep. 2002 }\end{array}$ & $\begin{array}{r}\text { Frame size } \\
\text { [pixel] }\end{array}$ & $N_{\mathrm{T}}$ & $N_{\mathrm{R}}$ & $\begin{array}{r}T \\
{[\mathrm{~ms}]}\end{array}$ & $\begin{array}{r}\text { Seeing } \\
{\left[{ }^{\prime \prime}\right]}\end{array}$ \\
\hline 22 & $192 \times 192$ & 280 & 1111 & 164 & 2.5 \\
23 & $192 \times 192$ & 368 & 529 & 164 & 2.4 \\
25 & $512 \times 512$ & 545 & 732 & 590 & 1.4 \\
\hline
\end{tabular}

between the outflowing gas and the parent molecular cloud. The remarkable details of the nebula of gas and dust expelled from the young star, revealed by these images, show that AFGL 2591 is a very interesting target for further, more detailed studies.

\section{Observations and data analysis}

The speckle interferograms of AFGL 2591 were recorded in September 2002 with the SAO 6 m telescope in Russia. The detector of our speckle camera was a Rockwell HAWAII array (only one $512 \times 512$ quadrant was used). The size of one pixel corresponds to 27 mas on the sky. A $K$-band filter with central wavelength $2115 \mathrm{~nm}$ and bandwidth $214 \mathrm{~nm}$ was used. Further observational parameters are listed in Table 1.

The modulus of the Fourier transform of the object (visibility) was obtained with the speckle interferometry method (Labeyrie 1970). The two small-field data sets from Sep. 22 and Sep. 23 were used to reconstruct the visibility of the central source in AFGL 2591. Speckle interferograms of the unresolved single star BD +394195 were recorded just before and after the object and served to determine the speckle transfer function.

An image with a resolution of 170 mas (Fig. 1) was reconstructed from the large-field data set observed on Sep. 25 using the bispectrum speckle interferometry method (Weigelt 1977; Weigelt \& Wirnitzer 1983; Lohman et al. 1983; Hofmann \& Weigelt 1986). For this image reconstruction, the central star speckle interferograms of AFGL 2591 itself were used for the determination of the speckle transfer function. For this purpose, we cutted out the inner $2^{\prime \prime}$ region of the central star speckle interferograms and derived from this data the transfer function.

\section{Results}

Our 170 mas resolution speckle reconstruction is presented in Fig. 1. In addition to the very bright, point-like source AFGL 2591 (see Sect. 3.1), our data show extended diffuse nebulosity (see Sect. 3.2) and several fainter point sources, including a faint $0.5^{\prime \prime}$ separation binary located about $4^{\prime \prime}$ to the West of AFGL 2591. We note that the bright source AFGL 2591 is not saturated in our raw data and reconstructions, although it is much brighter than the other features visible in our large-field image: for example, it is 7.3 mag brighter than the north-western component of the close binary to the West and $\sim 8.6$ mag brighter than the star $\sim 5^{\prime \prime}$ North of it. Due to the enormous brightness contrast in the image, the diffraction rings and the noise pattern in the wings of the AFGL 2591 image appear very prominently. 


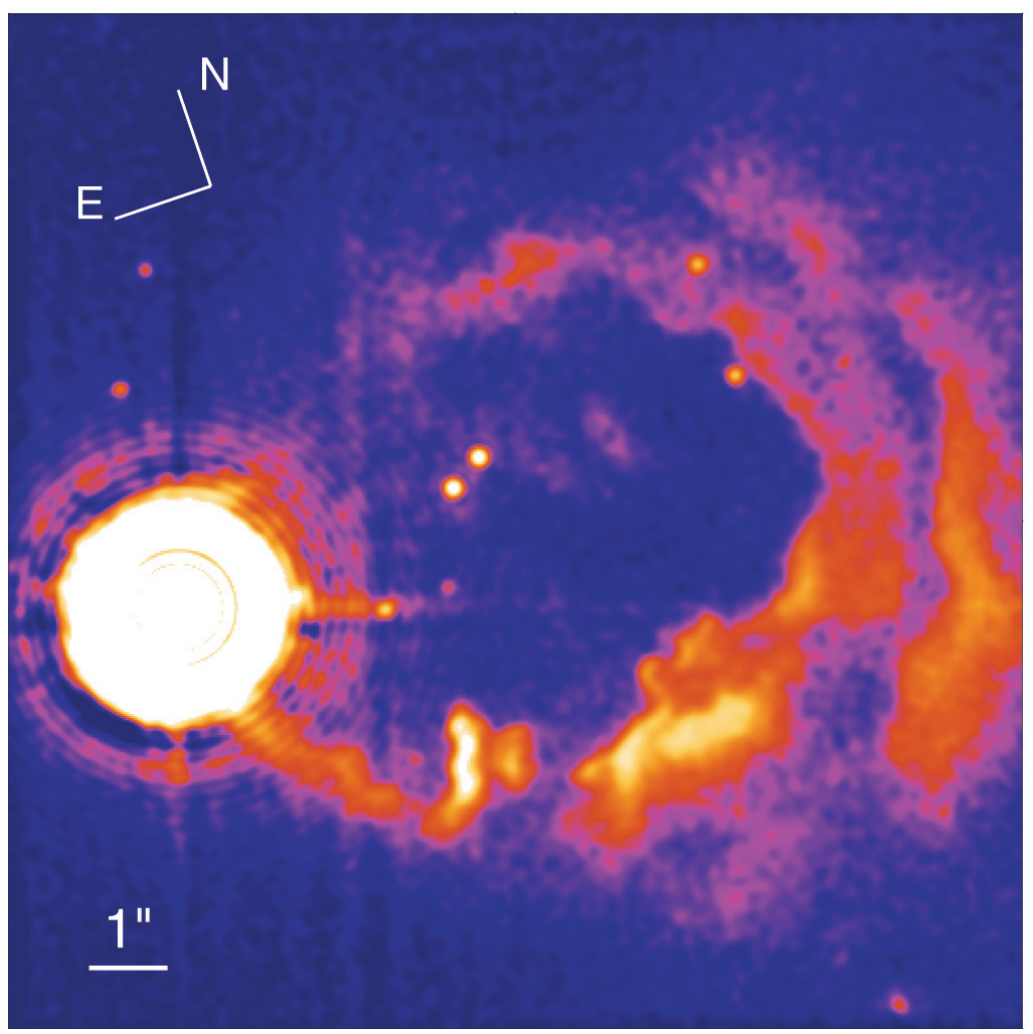

Fig. 1. Pseudocolor representation of our large-field $K$ band image of AFGL 2591, reconstructed with the bispectrum speckle interferometry method by using AFGL 2591 itself as a reference star. This image has a resolution of 170 mas and shows a $\sim 13^{\prime \prime} \times 13^{\prime \prime}$ field of view. The directions to North and East are indicated. In order to show the faint loop-like structures with good contrast, the intensity scale only covers intensities up to $0.2 \%$ of the peak. The bright source is not saturated; since it is much brighter than the other features in the image ( $~ 8.6$ mag brighter than the star $\sim 5^{\prime \prime}$ North of it) it is surrounded by prominent wings in this image.

\subsection{The central source}

The visibility (Fig. 2) shows that the central source is clearly extended. The drop of the visibility, however, is too small to derive detailed information about the shape of the resolved structure. A fit to the visibility function with a Gaussian disk model yields an FWHM diameter of $24 \pm 4$ mas. This corresponds to physical scales of about 24 AU. A fit with a uniform-disk model gives a diameter of $39 \pm 7$ mas, and a two-component Gaussian disk model yields diameters of $\sim 22 \mathrm{AU}$ for the small component and $\sim 260$ AU for the large component. A detailed interpretation of the observed source size will be given in Sect. 4.2. We note that the visibility is not perfectly symmetric. The deviations from radial symmetry, however, are too small and uncertain for an analysis of the shape; we therfore consider only azimuthally averaged visibilities.

\subsection{The nebulous loops}

The nebulosity in our image is dominated by two nonconcentric elongated loops that seem to originate from the bright compact source. In the following text we will denote the inner loop seen in our speckle image as "loop I" and the outer loop as "loop II". The length of the major axis of these loops are $8.8^{\prime \prime}$ for loop I and 10.3" for loop II. While the general shape of the loops is quite well defined, both show a very clumpy structure.

We believe that these loops represent the limb-brightened flanks of elongated bubbles that have been created by material flowing away from AFGL 2591. Therefore, we compare their observed shapes with the model for propagating outflows presented by Gueth \& Guilloteau (1999). These authors presented semi-analytical models of jet-driven flows, in which prompt entrainment occurs at the head of the traveling bow shock, with which they could reproduce the observed shape of the cavity of to the HH 211 jet very well.

Projected shapes computed with this model are over-plotted on our speckle image in Fig. 3. The model fits the observed shapes of the loops quite well. It is obvious that the loops are very broad and not strongly collimated. To put this argument into a more quantitative way, we note that the collimation factor, which is defined as the ratio $d / R_{0}(d)$ and gives the ratio between the characteristic length and the characteristic width of the loops, is 3.5 for loop I and 2.85 for loop II. Well collimated outflows, like HH 211, typically have collimation factors above 20 (Gueth \& Guilloteau 1999). The fits with the theoretical model also allow us to determine the position angle of the loop axis. Our fits give $\psi=265^{\circ} \pm 1^{\circ}$ for loop I and $\psi=263^{\circ} \pm 1^{\circ}$ for loop II.

The Gemini image shows two additional loops further out, which we will call "loop III" and "loop IV", and for which we determined diameters of $21^{\prime \prime}$ and $28^{\prime \prime}$, respectively. The general shape of these outer loops can also be well fitted by the outflow model. These fits give collimation factors of 7 and position angles of $\psi=262^{\circ} \pm 1^{\circ}$ for loop III and loop IV. It is interesting to note that there seems to be a systematic change in the loop orientation from $\psi=265^{\circ}$ for loop I to $\psi=262^{\circ}$ for loop IV. If the loop system was created in a sequence of ejection events, the direction of the ejection axis has apparently moved counter-clock wise.

Finally, we note that the inner two loops appear very symmetric, whereas loop IV shows some kind of depression in its 

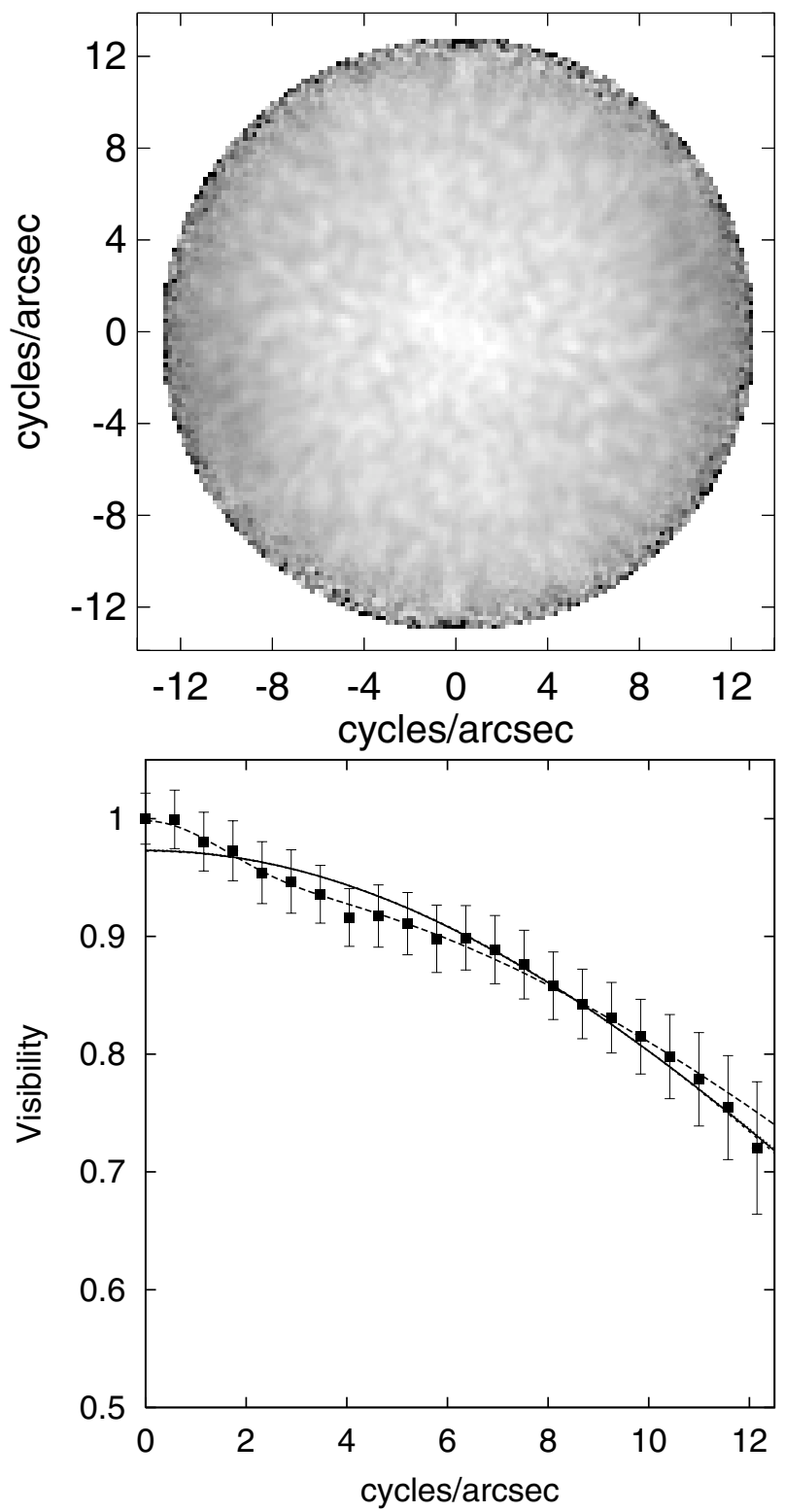

Fig. 2. Top: reconstructed two-dimensional visibility of the bright compact source in AFGL 2591. Bottom: radial dependence of the azimuthally averaged $K$-band visibility of AFGL 2591 (boxes with error bars). The plot also contains several model visibilities: the visibility predicted from our radiation transfer simulation for the disk model (see Sect. 4.2 for further information) is shown as a solid line. The visibilities for the Gaussian disk model and the uniform-disk model are plotted as dotted and dashed-dotted lines, respectively, but are not visible since they are almost identical to the solid line. The dashed line shows the two-component Gaussian disk model.

southern part. This might trace the interaction of the expanding loops with the ambient material, which causes deformations in the initially symmetric loops.

\subsection{Possible edge-on disk}

In the southern part of loop I, our image shows a prominent feature which consists of two elongated, bended structures, oriented roughly in north-south direction (see mark in Fig. 3).

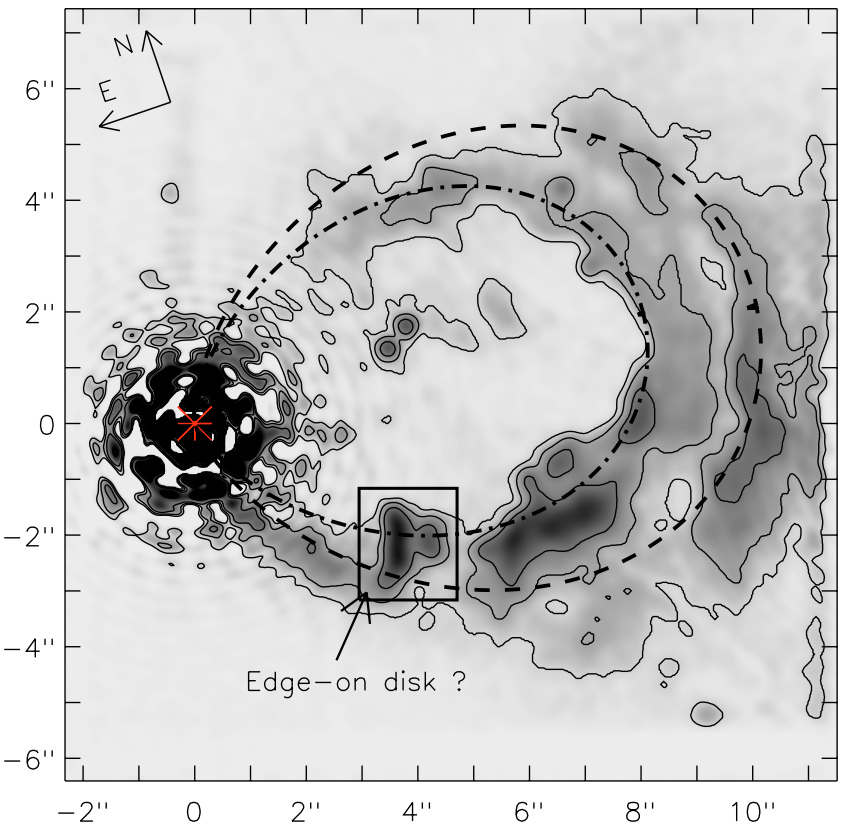

Fig. 3. Greyscale + contour representation of our $K$-band speckle image reconstructed with a reduced resolution of 280 mas in order to show more clearly the fainter details. The analytic models for loop I (the smaller loop) and loop II (the larger loop) are overplotted by the thick dashed lines. The possible edge-on disk is marked.

This feature, for which an enlarged and rotated image is shown in Fig. 4, has a remarkable similarity to a circumstellar disk seen close to edge-on, in which the direct view of the star is blocked, while a small amount of the stellar light is scattered below and above the disk midplane, producing a dark lane between two reflection nebulae. High-spatial resolution observations have revealed several edge-on disks in different star forming regions (e.g. Burrows et al. 1996; Stapelfeldt et al. 1998; Brandner et al. 2000; Monin \& Bouvier 2000; Grosso et al. 2003) which look very similar to the structure in our image. The difference in brightness between the eastern and western reflection lobes can be explained as an inclination effect. With a radius of about $0.9^{\prime \prime}$ or $900 \mathrm{AU}$ and a thickness of the dark lane of about $0.24^{\prime \prime}$ or $240 \mathrm{AU}$, the dimensions of the feature are in the typical range found for other edge-on disks.

Of course, our image does not provide clear proof that this feature actually is a disk; it could just be a random structure that is illuminated by AFGL 2591. However, this feature is at least a very good edge-on disk candidate and deserves more detailed observations.

\section{Interpretation}

\subsection{The outflow activity of AFGL 2591}

While the presence of the loops around AFGL 2591 has been noted in previous papers, our high-resolution images have allowed a more detailed investigation of their structure and orientation. In combination with previous results, we now consider all observed outflow tracers (i.e. the loops, the $\mathrm{H}_{2}$ bows, and the HH objects) to investigate the outflow activity of AFGL 2591. 


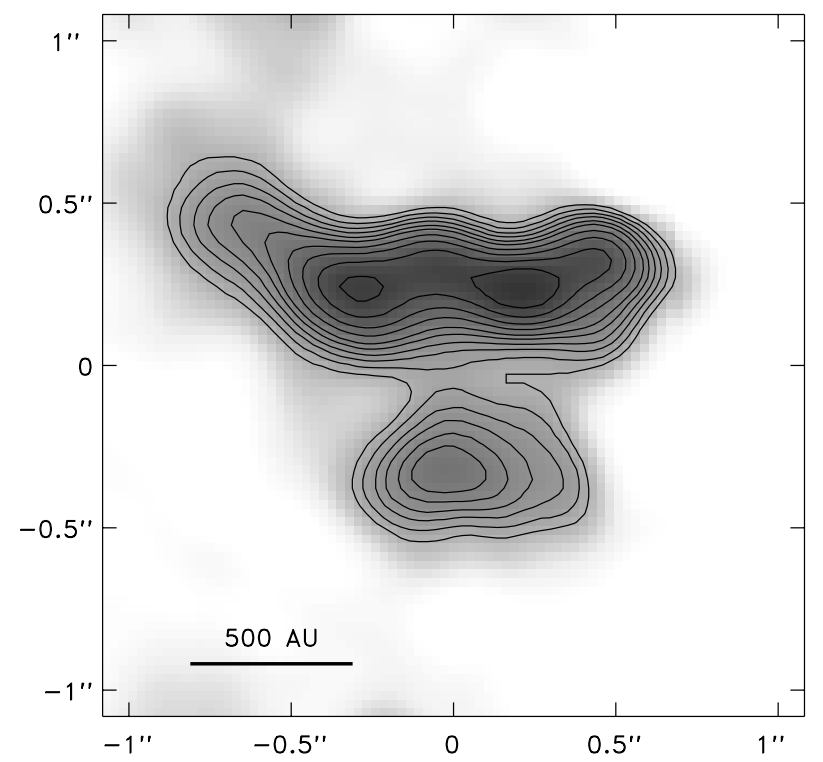

Fig. 4. Greyscale + contour representation of our image of the suspected edge-on disk (see Sect. 3.3). The image was rotated in order to place the midplane of the structure along the $x$-axis.

\subsubsection{The driving mechanism of the observed flows}

Several models have been proposed for the mechanism by which jets and outflows from young stellar objects are created. In the jet-driven outflow model, a highly collimated jet with a bow shock at its head propagates into the ambient medium (see e.g. Suttner et al. 1997; Smith et al. 1997; Lee et al. 2001). The molecular outflow is produced as the jet carves into the cloud and entrains ambient gas. In the wide-angle wind model, the outflow is produced by the interaction of the stellar wind with the ambient material, which is swept up into a shell by the shock at the wind/cloud interface (see e.g. Shu 1991; Lee et al. 2001).

AFGL 2591 displays quite different manifestations of outflow activity: the apparently well collimated, jet-like flows that created the $\mathrm{HH}$ objects and the $\mathrm{H}_{2}$ bows, as well as a wideangle flow that created the broad loops and carved out the rather wide cavity. The most straightforward explanation for the presence of the wide loops would be the action of a strong stellar wind from the central protostar. The initially spherical stellar wind is, to some degree, collimated by the dense, flattened structure (perhaps a circumstellar disk) around the protostar, and the loops can naturally be explained as wind-bubbles, which are created as the wind pushes against the environment and sweeps up the ambient material. The presence of four individual loops suggests that the wind is variable in time; the sequence of four loops then traces a sequence of four periods of increased wind power, or outbursts. We can estimate the dynamical ages of the loops by assuming a wind velocity of $v_{\text {wind }}=500 \mathrm{~km} \mathrm{~s}^{-1}$ (van der Tak et al. 1999); for an inclination angle of $i=30^{\circ}$, the diameters of the loops then suggest dynamical ages of between $175 \mathrm{yrs}$ and $500 \mathrm{yrs}$, with time intervals between the individual outbursts of about 50-200 years. As the winds from massive stars are radiation-driven, the periods of increased wind power probably correspond to periods of increased luminosity. One possible explanation might be increased accretion activity, as suspected, for example, in the case of the strongly variable FU Orionis stars. The derived timescales are in fact in a similar range as the activity observed in FU Ori stars (e.g. Hartmann \& Kenyon 1996). The multiple loops can therefore be easily explained as being caused by a strong and variable stellar wind.

How are the $\mathrm{HH}$ objects and the $\mathrm{H}_{2}$ bows, which apparently require highly collimated, jet-like flows, related to the loops? Are there two, fundamentally different driving mechanisms simultaneously at work in AFGL 2591, i.e. a strong, wide-angle stellar wind and highly collimated, jet-like flows, or can all observed outflow phenomena be understood in a single, unified model?

Possibility I: Purely wind driven flows? We will first consider the question whether a wide-angle stellar wind model might be able to explain all the different manifestations of outflow activity observed in AFGL 2591. While the broad western loops are easily explained as being wind-blown bubbles, it might appear difficult for a wide-angle wind to create the eastern bows. However, we note that the group of bows East of AFGL 2591 displays a very complex morphology: the Gemini image suggests the presence of numerous (at least 5) individual bows, which seem to propagate in significantly different directions, covering at least $20^{\circ}$. Canonical jet models, however, predict only a single, usually straight jet; several bow shocks are observed in some jets, but the individual bows are usually well aligned and point all in the same direction, in strong contrast to the case of the AFGL 2591 bows. A single, time variable jet can, in principle, create multiple bows with different orientations, if the jet direction is variable, for example, due to precession, or if the jet interacts with and is deflected at ambient cloud material (e.g. Reipurth \& Bally 2001). However, these mechanisms would be expected to create a pattern of systematic changes in the jet direction (see e.g. the simulations in Masciadri et al. 2002), whereas the bow complex to the East of AFGL 2591 appears as if individual bullets have been nearly simultaneously ejected in different directions. An alternative explanation for the morphology of the bow complex to the East of AFGL 2591 might be the time-variable wind model by Stone et al. (1995). This model was developed to explain the structure of the spectacular outflow from the massive protostar in the Orion BN-KL complex, which consists of dozens of bow-shocks (so-called "fingers") that cover a very wide angle on the sky (see, e.g., Salas et al. 1999). The model by Stone et al. (1995) assumes that the wind from the protostar creates an expanding wind bubble. The surface of the wind-blown shell is initially smooth and homogeneous. If, however, the wind velocity increases strongly, the wind-blown shell is accelerated and can become Rayleigh-Taylor instable. The shell then breaks up into numerous fragments, each of which produces a bow shock as it plows through the ambient medium. This model might offer a good explanation for the observed morphology of the bows East of AFGL 2591. Furthermore, the presence of the four loops around AFGL 2591 provides independent evidence for temporal variability of the stellar wind and therefore also 


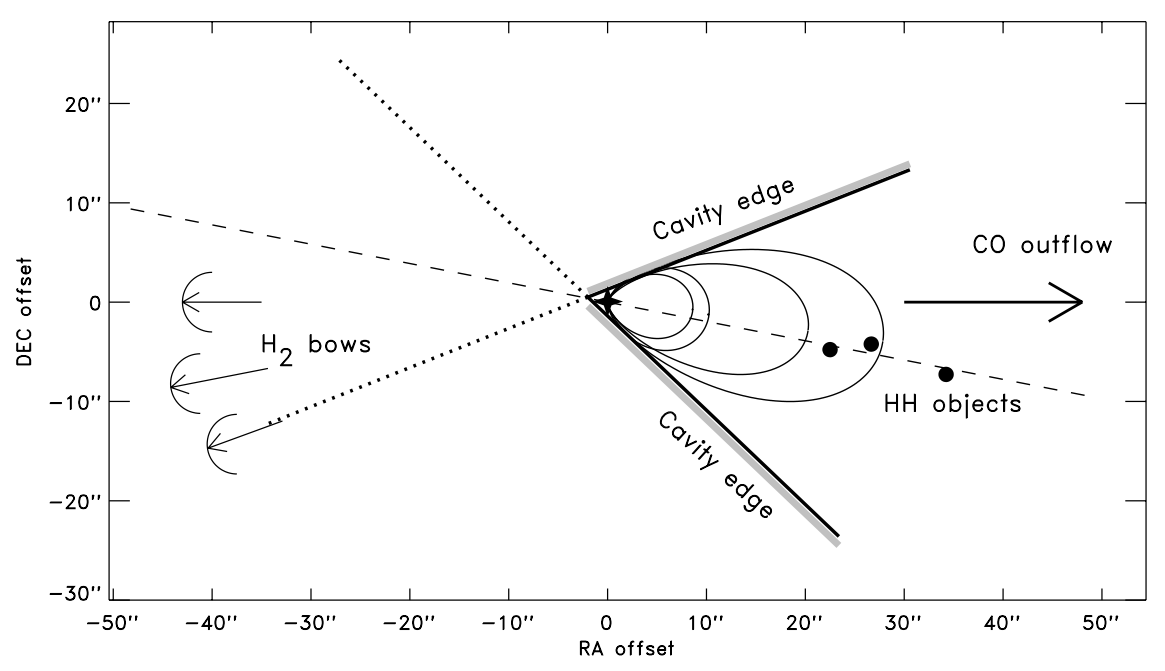

Fig. 5. Sketch of the system and the different outflow tracers. The edges of the cavity, the shape of the loops to the West of AFGL 2591, the direction of the blue-shifted $\mathrm{CO}$ outflow, and the location of the $\mathrm{HH}$ objects and the eastern bow shocks are shown. The long dashed line shows the main axis of the western outflow tracers and the cavity. supports this model. In the frame of the time variable wind model, the loops would be the most recently ejected wind bubbles, whereas the multiple bows to the East are parts of a previously ejected bubble that has already fragmented.

Possibility II: Purely jet driven flows? Is it possible that the broad western loops have been created by jet-like flows? The quite regular shape of the loops makes it difficult to imagine how they could have been created by a narrow jet flow. However, we note that some highly collimated jets create very broad bow shocks with much larger opening angles than the jet (see for example Reipurth et al. 2002 for the case of $\mathrm{HH}$ 34). Also, it is known that meandering jets can eventually produce wide outflow cavities and drive poorly collimated outflows by the combined action of jet direction variations (e.g. due to precession) and velocity variation (Reipurth \& Bally 2002). Therefore, the alternative unified model, in which all outflow phenomena are ultimately caused by highly collimated jet-like flows, appears also to be a valid possibility.

We finally note that, since AFGL 2591 is a rather massive $\left(\sim 10-15 M_{\odot}\right)$ protostar and since stellar luminosity and therefore wind power both increase very strongly with stellar mass, we believe that the stellar wind of AFGL 2591 plays an important role in driving the outflows. The flows probably derive at least some of their power from radiation pressure, and ionizing radiation might play an important role too (see discussion in Reipurth \& Bally 2002).

\subsubsection{The outflow directions}

Another interesting aspect of the observed outflows from AFGL 2591 concerns the flow directions. On the western side, the directions of the loops (PA $265^{\circ}-262^{\circ}$ ) are well consistent with the direction of the blue-shifted $\mathrm{CO}$ outflow (PA $\sim 270^{\circ}$; Yamashita et al. 1987), the position angles of the Herbig-Haro objects $\left(\mathrm{PA}=258^{\circ}\right.$ to $\left.261^{\circ}\right)$, and the orientation of the cavity (with its symmetry axis along PA $=259^{\circ}$; see the scetch in Fig. 5). Therefore, the directions of all outflow tracers to the West of AFGL 2591 agree very well and suggest that the current outflow direction towards the West is within $\pm 10^{\circ}$ of $\mathrm{PA}=260^{\circ}$.
The bows to the East of AFGL 2591, however, are not found along the opposite direction of the western outflow indicators, which would be $80^{\circ}$, but rather span a range of position angles from $90^{\circ}$ to $110^{\circ}$, the mean axis of the bow shock system and the most prominent shock being at $\mathrm{PA} \sim 100^{\circ}$. The mean direction of the $\mathrm{H}_{2}$ outflow is therefore tilted by $20^{\circ}$ from the counter-direction of the western flows. This difference is remarkable, but we note that the observed range of directions of the $\mathrm{H}_{2}$ bow shocks is still within the opening angle of the cavity. As the $\mathrm{H}_{2}$ bow shocks are further away from the central source than the loops and the $\mathrm{HH}$ objects on the western side, they must have been ejected earlier. This might imply that the outflow axis has changed with time.

\subsubsection{Dynamics of the loops}

Given the measured flow velocities in the AFGL 2591 system (e.g. van der Tak et al. 1999), we expect the loops to expand at rates of the order of a few $100 \mathrm{~km} \mathrm{~s}^{-1}$. Expansion velocities in the range $100-500 \mathrm{~km} \mathrm{~s}^{-1}$ would correspond to projected expansion rates of 10-50 mas/yr for an inclination angle of $i=30^{\circ}$. By repeating our speckle observations, we would therefore expect to be able to trace the expansion of the loops within a few years.

We also have carefully compared the Gemini image, which was obtained in 2001, with our speckle image, and found no significant differences in the diameters of the two inner loops. However, due to the differences in spatial resolution of the Gemini image and our speckle image, the accuracy of our comparison is not better than about 200 mas, whereas the expected change in diameter is $\$ 50$ mas.

\subsection{The nature of the central source}

We believe that the uniform-disk diameter of $\sim 40$ mas of the central source is closely related to the dust sublimation radius that defines the inner rim of the circumstellar material around the protostar. In order to get an appropriate estimate of the dust sublimation radius and to find out what kind of structure one would expect to see, we have performed 
radiation transfer calculations. As the circumstellar environment of AFGL 2591 is obviously not spherically symmetric, simple 1D radiation transfer programs cannot be applied. For our simulations we therefore used the $2 \mathrm{D}$ radiation transfer code described in Sonnhalter et al. (1995). In this code, the distribution of dust temperatures and radiation intensities in an axially symmetric dusty circumstellar environment around a central radiation source is calculated within the framework of the flux-limited diffusion approximation (Levermore \& Pomraning 1981). The equations are discretized on a quadratic, equally spaced grid. To improve resolution and convergence, a system of 5 nested grids with decreasing grid spacing is used. The differential equations for the radiation field are iterated together with the temperature equations until a self-consistent equilibrium configuration is reached. After determination of the dust temperatures for a multi-component dust model, a ray-tracing procedure is used to calculate intensity maps for the appearance of the central object and its circumstellar environment at varying inclinations for selected frequencies. A detailed description of the code is given in Sonnhalter et al. (1995).

In our simulations we assume a luminosity of $20000 L_{\odot}$ and an effective temperature of $30000 \mathrm{~K}$ for the central source, as suggested by van der Tak et al. (1999). We use a dust model from Preibisch et al. (1993), which was constructed to reproduce the dust properties in dense molecular clouds. It consists of small amorphous carbon grains and large silicate grains and assumes that the silicate grains are coated with a mantle of "dirty ice" if their temperature is below $125 \mathrm{~K}$. The radii $a$ of the grains obey a power law distribution of the form $n(a) \propto a^{-3.5}$, where we assume that the carbon grain radii range from $7 \mathrm{~nm}$ to $30 \mathrm{~nm}$ and the silicate radii from $40 \mathrm{~nm}$ to $1 \mu \mathrm{m}$. The ratio between the radii of ice-coated and uncoated silicate grains is 1.145 . The "dirty ice" consists of a 3:1 (volume) mixture of $\mathrm{H}_{2} \mathrm{O}$ and $\mathrm{NH}_{3}$, which is polluted with $10 \%$ carbon particles. When the ice coatings sublimate, the carbon particles are set free and a "naked" silicate grain remains. The sublimation temperatures of the silicate and carbon particles are taken to be $1500 \mathrm{~K}$. The destruction of grains through sublimation is simulated by setting the number density of the grains to zero, if the temperature of a particular grain component calculated simultaneously with the radiation field is above the sublimation temperature. The inner boundary of the dusty environment around the central source is therefore given by the point of dust sublimation.

We consider two different models for the density distribution of the circumstellar material. Our first model assumes that the central source is embedded in a dusty envelope with a radial power-law density distribution extending out to $30000 \mathrm{AU}$ (see van der Tak et al. 1999). The cavities are simulated as a pair of cones with their tips at the position of the central star, in which the density is 1000 times smaller than in the other parts of the envelope at the same radial distance from the center. The model is given by:

$\rho_{\text {envelope }}= \begin{cases}\rho_{0} \times\left(\frac{r}{r_{0}}\right)^{-1.25} & \text { for } r / z \geq 0.554 \\ \frac{1}{1000} \times \rho_{0} \times\left(\frac{r}{r_{0}}\right)^{-1.25} & \text { for } r / z<0.554\end{cases}$ where $\rho_{0}$ is the density of the envelope at a characteristic radius $r_{0}=2.5 \times 10^{15} \mathrm{~cm}=167 \mathrm{AU}$. The full opening angle of the cavities is $2 \times \arctan (0.554)=58^{\circ}$.

Our second model assumes a geometrically thick Keplerian disk, which is given by:

$\rho_{\text {disk }}=\rho_{0}\left(\frac{r_{0}}{r}\right)^{15 / 8} \mathrm{e}^{-\pi / 4\left(z / z_{0}\left(r_{0} / r\right)^{9 / 8}\right)^{2}}$.

The parameters $r_{0}$ and $z_{0}=h r_{0}$ fix the radius and height of the disk, and $\rho_{0}$ is the density of the disk at a characteristic radius $r_{0}=2.5 \times 10^{15} \mathrm{~cm}=167 \mathrm{AU}$ and $z=0$. For $h=0.7$, this density distribution yields a thick disk which is embedded in a torus-like envelope with low-density cones along the polar axis with a full opening angle of $\sim 65^{\circ}$.

Simulated $K$-band images for our two models, assuming an inclination angle of $i=30^{\circ}$ as derived by van der Tak et al. (1999), are shown in Fig. 6. In both cases, the dominant structure is the bright inner wall of the dusty envelope or disk at the dust sublimation radius. The size of the bright structure is $\sim 38 \times 31 \mathrm{AU}$ in the envelope/cavity model and $\sim 34 \times 24 \mathrm{AU}$ in the disk model. We emphasize that small changes of the model parameters do not affect the simulated images noticeably. We also used our radiation transfer simulations to compute the expected visibilities and compare them to the measured visibility for AFGL 2591. A comparison of the disk model simulation to the observed data is shown in Fig. 2 and shows generally good agreement. The envelope/cavity model predicts a very similar visibility and is therefore not shown.

As described above, the observed visibility of the source can also be well fitted with a simple uniform-disk model, and the resulting diameter is $\sim 39 \mathrm{AU}$. This uniform-disk fit diameter is in good agreement with the size of the bright structures in our simulated images and supports our interpretation of the resolved structure as the dust sublimation wall around the central star. Since the uniform-disk model gives an essentially equally good fit to the observed visibility as our two simulated model images, the observed visibility does not allow us to derive detailed information about the shape of the resolved structure.

At a closer look, the measured visibility of AFGL 2591 shows some deviations from the model curve and seems to have a more complex structure. This probably reflects the fact that the true geometry of the source is more complex than the rather simple density models used in our simulations. However, it is not our aim here to perform a detailed fit of the observed data with sophisticated models, what is hardly possible given the available data. Our simulations are just thought as an illustration of how the inner circumstellar environment of the protostar might look like and can explain the observed size quite well.

Finally, we note that the uncertainty in the distance of AFGL 2591 does not affect our interpretation of the resolved structure as the dust sublimation radius: if the distance to the star were actually larger, its estimated luminosity (which was based on the assumed distance) would increase as $L_{\star} \propto D^{2}$; since the dust sublimation radius scale approximately as $R_{\text {sub }} \propto$ $\sqrt{L}_{\star}$, the angular size of the dust sublimation radius $R_{\text {sub }} / D$ stays constant.

We note that the observed size of AFGL 2591 is also well consistent with interferometric observations of other young 

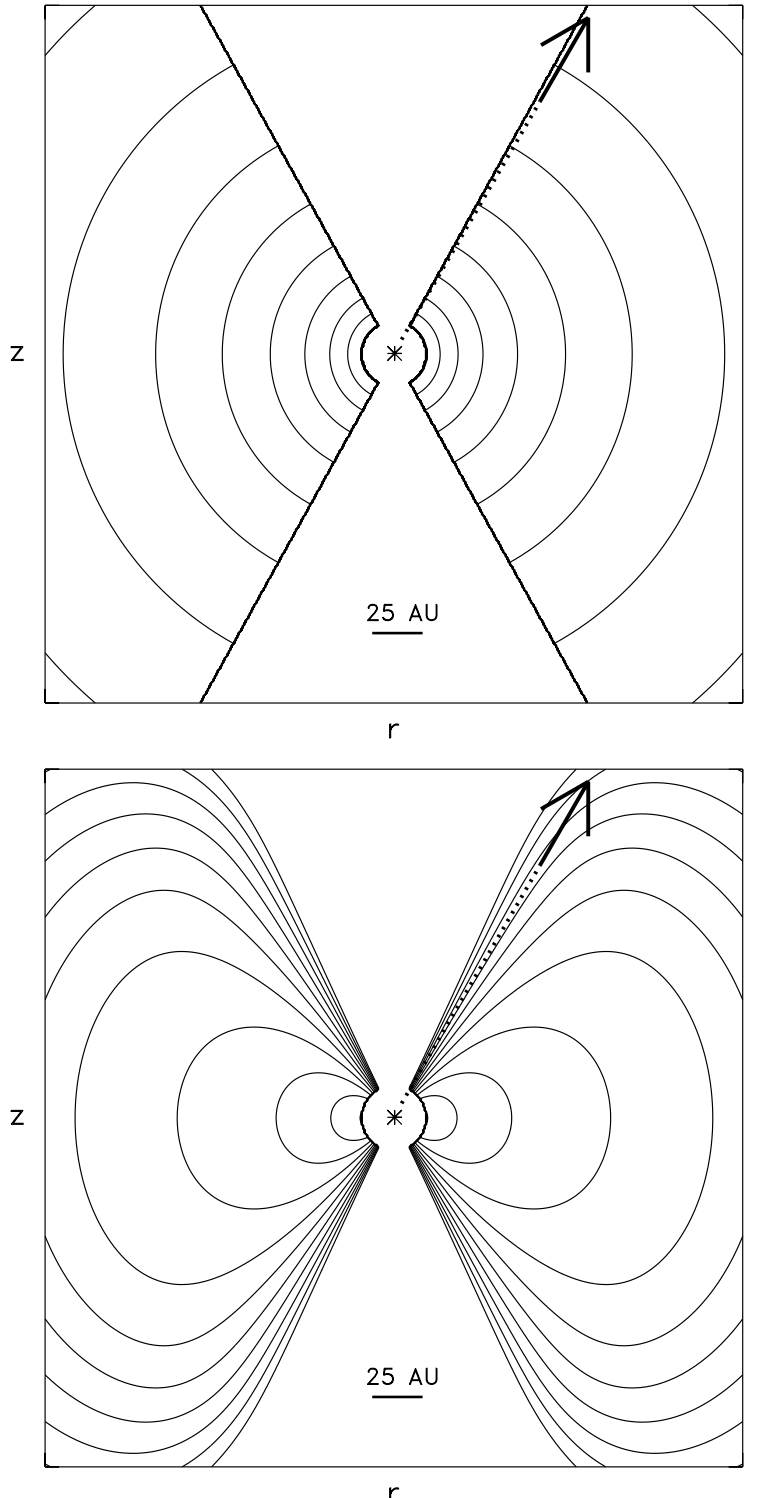
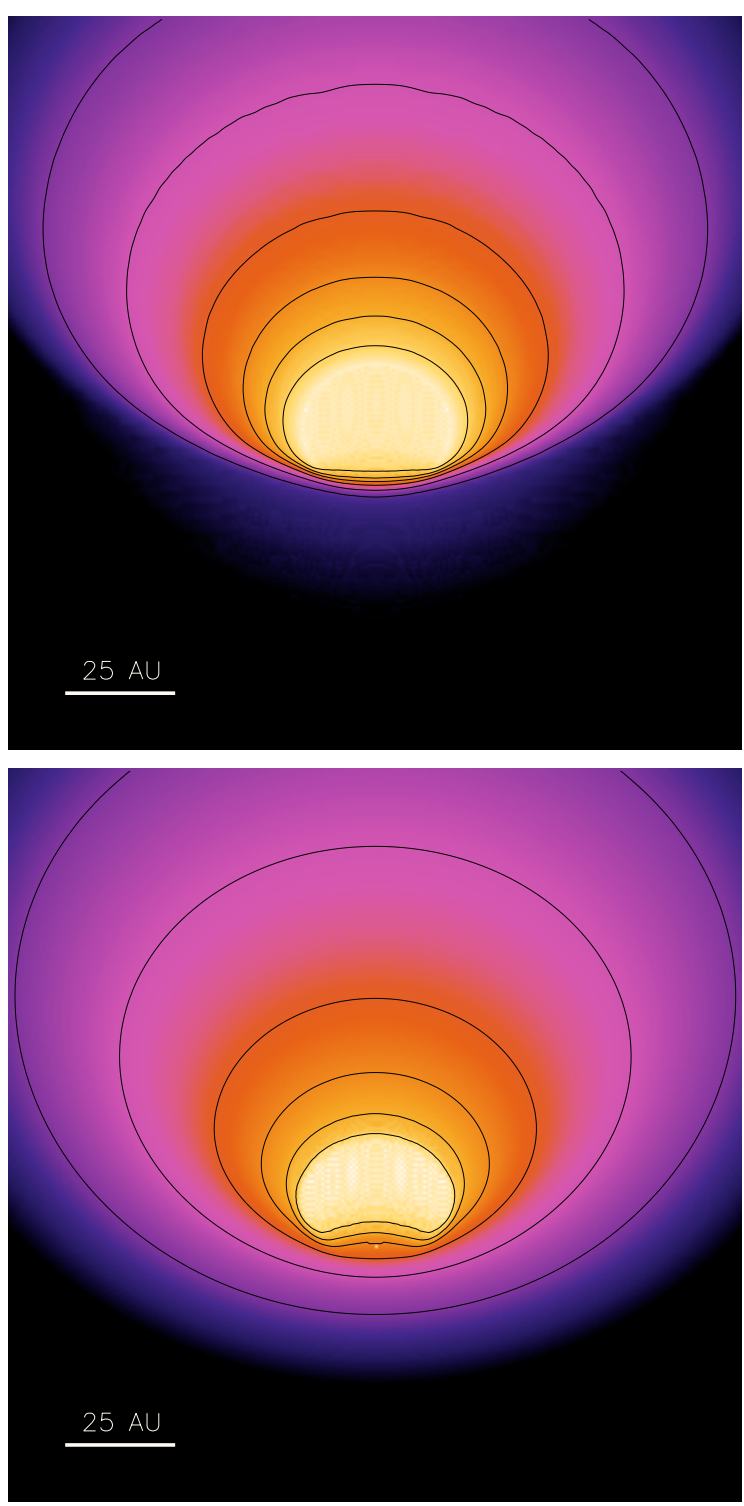

Fig. 6. Left column: contour representation of cuts in the central $r-z$-plane for the two density models used for the radiation transfer calculations, the envelope/cavity model (above; contour lines within the cavity are not shown) and the disk model (below). The innermost contour line shows the dust sublimation wall at a radius of $16 \mathrm{AU}$. The contour levels are logarithmically equidistant with 0.25 dex steps for the envelope/cavity model and 0.5 dex for the disk model. The dashed lines with the arrows show the direction towards the observer $\left(i=30^{\circ}\right)$. Right column: $K$-band model images resulting from the radiation transfer simulations for an inclination angle of $i=30^{\circ}$. The envelope/cavity model is shown in the upper plot, the disk model in the lower plot. A logarithmic intensity scale is used. The contours are drawn at levels of $0.7,1.5,2,3$, and 4 dex below the peak intensity. In both cases, the bright white/yellow structure is the inner wall of the dusty envelope or disk at the dust sublimation radius.

stellar objects. Monnier \& Millan-Gabet (2002) summarized recent long-baseline interferometric observations of young stellar objects and argued that the near-infrared sizes being measured are closely related to the dust sublimation radii. They fitted the interferometric visibilities with simple ring models and found typical inner ring radii ranging from about $0.1 \mathrm{AU}$ for low-luminosity $\mathrm{T}$ Tauri stars up to about $10 \mathrm{AU}$ for luminous Herbig Ae/Be stars. For the luminosity of AFGL 2591 $\left(20000 L_{\odot}\right)$, their models predict inner ring radii of about 710 AU. This is in good agreement with the size we derived for AFGL 2591 from our speckle data, i.e. a radius of $\sim 12$ AU. AFGL 2591 seems therefore to fit well into the general relation between size and luminosity presented in Monnier \& MillanGabet (2002).

\section{Summary and conclusions}

Our high-resolution $K$-band image shows the clumpy structure of the inner loops of the AFGL 2591 outflow in unprecedented detail. By repeating the observations of AFGL 2591 we can expect to trace the expansion of the loops within a few years. This will help to understand the mechanism by which the loops were created and to get more insight into the physical nature of the outflows of AFGL 2591. We have shown that the resolved 
40 AU sized central structure of AFGL 2591 can be understood as the inner wall of the circumstellar material at the dust sublimation radius. The possible edge-on disk near AFGL 2591 also deserves further observational efforts.

\section{References}

Bally, J., \& Lada, C. J. 1983, ApJ, 265, 824

Bally, J., \& Reipurth, B. 2001, ApJ, 546, 299

Burrows, C. J., Stapelfeldt, K. R., Watson, A. M., et al. 1996, ApJ, 473,437

Brandner, W., Sheppard, S., Zinnecker, H., et al. 2000, A\&A, 364, L13

Dougados, C., Cabrit, S., Lavalley, C., \& Menard, F. 2000, A\&A, 367, L61

Eislöffel, J., Mundt, R., Ray, T. P., \& Rodriguez, L. F. 2000, in Protostars and Planets IV, ed. V. Mannings, A. P. Boss, \& S. S. Russel, (Tucson: University of Arizona Press), 815

Grosso, N., Alves, J., \& Wood, K. 2003, ApJ, 586, 296

Hartmann, L., \& Kenyon, S. J. 1996, ARA\&A, 34, 207

Hodapp, K.-W. 1994, ApJS, 94, 615

Labeyrie, A. 1970, A\&A, 6, 85

Lada, C. J., Thronson, H. A., Smith, H. A., Schwartz, P. R., \& Glaccum, W. 1984, ApJ, 286, 302

Lee, C.-F., Stone, J. M., Ostriker, E. C., \& Mundy, L. G. 2001, ApJ, 542,925

Levermore, C. D., \& Pomraning, G. C. 1981, ApJ, 248, 321

Lohmann, A. W., Weigelt, G., \& Wirnitzer, B. 1983, Appl. Opt., 22, 4028

Masciadri, E., De Gouveia Dal Pino, E. M., Raga, A. C., \& NoriegaCrespo, A. 2002, ApJ, 580, 950

Minchin, N. R., Hough, J. H., McCall, A., et al. 1991, MNRAS, 251, 508

Mitchel, G. F., Maillard, J.-P., \& Hasegawa, T. I. 1991, ApJ, 371, 342

Monin, J.-L., \& Bouvier, J. 2000, A\&A, 356, L75

Monnier, J. D., \& Millan-Gabet, R. 2002, ApJ, 579, 694

Poetzel, R., Mundt, R., \& Ray, T. P. 1992, A\&A, 262, 229
Preibisch, Th., Ossenkopf, V., Yorke, H. W., \& Henning, Th. 1993, A\&A, 279, 577

Preibisch, Th., Sonnhalter, C., \& Yorke, H. W. 1995, A\&A, 299, 144

Preibisch, Th., Balega, Y. Y., Schertl, D., Smith, M. D., \& Weigelt, G. 2001, A\&A, 378, 539

Preibisch, Th., Balega, Y., Schertl, D., \& Weigelt, G. 2002, A\&A, 392, 945

Reipurth, B., \& Bally, J. 2001, ARA\&A, 39, 403

Reipurth, B., Heathcote, S., Morse, J., Hartign, P., \& Bally, J. 2002, AJ, 123, 362

Salas, L., Rosado, M., Cruz-Gonzales, I., et al. 1999, ApJ, 511, 822

Schertl, D., Balega, Y., Hannemann, T., et al. 2000, A\&A, 361, L29

Shu, F. H., Ruden, S. P., Lada, C. J., \& Linzano, S. 1991, ApJ, 370, L31

Smith, M. D. 1993, ApJ, 406, 520

Smith, M. D., Suttner, G., \& Yorke, H. W. 1997, A\&A, 323, 223

Sonnhalter, C., Preibisch, Th., \& Yorke, H. W. 1995, A\&A, 299, 545

Stapelfeldt, K. R., Krist, J. E., Menard, F., et al. 1998, ApJ, 502, L65

Stone, J. M., Xu, J., \& Mundy, L. G. 1995, Nature, 377, 315

Suttner, G., Smith, M. D., Yorke, H. W., \& Zinnecker, H. 1997, A\&A, 318,595

Tamura, M., Gatley, I., Joyce, R. R., et al. 1991, ApJ, 378, 611

Tamura, M., \& Yamashita, T. 1992, ApJ, 391, 710

Weigelt, G. 1977, Opt Commun., 21, 55

Weigelt, G. 1991, in Progress in Optics, 29, ed. E. Wolf (Elsevier Science Publishers), 295

Weigelt, G., Preibisch, Th., Schertl, D., Balega, Y. Y., \& Smith, M. D. 2002a, A\&A, 381, 905

Weigelt, G., Balega, Y., Hofmann, K.-H., \& Preibisch, Th. 2002, 2002b, A\&A, 392, 937

Van der Tak, F., Dishoeck, E. F., Evans II, N. J., \& Bakker, E. J. 1999, ApJ, 522, 991

Yamashita, T., Sato, S., Tamura, M., et al. 1987, PASJ, 39, 809

Yorke, H. W. 1980a, A\&A, 85, 215

Yorke, H. W. 1980b, A\&A, 86, 286

Yorke, H. W. 1985, in Birth and Infancy of Stars, ed. R. Lucas, A. Omont, \& R. Stora (Elsevier Science Publishers), 645 